\title{
¿Influye el estilo parental percibido por el hijo en el sobrepeso u obesidad infantil?
}

\section{Does parenting style perceived by the child influence childhood overweight or obesity?}

\author{
Ilse Tatiana Mejía Galván y Ferrán Padrós Blázquez ${ }^{2}$ \\ Secretaría de Educación del Estado de Michoacán ${ }^{1}$ \\ Universidad Michoacana de San Nicolás de Hidalgo ${ }^{2}$
}

Autor para correspondencia: Ilse Tatiana Mejía Galván,itmg_0112@hotmail.com.

\begin{abstract}
RESUMEN
Introducción: El aumento del sobrepeso y de la obesidad infantil se ha ido convirtiendo en un problema mundial en el ámbito de la salud física y psicológica. Este inconveniente tiene múltiples causas, entre las que se podría incluir el estilo parental. Objetivo: Conocer las posibles relaciones entre el estilo que los padres adoptan para educar a sus hijos y el sobrepeso y obesidad infantil de estos. Método: Las variables a estudiar fueron medidas a través de la Escala de Afecto (EA) y la Escala de Normas y Exigencias, suministradas a los niños y adolescentes. Dichas pruebas fueron aplicadas a 149 participantes de entre 12 y 16 años de edad, de los cuales 24 tenían sobrepeso u obesidad. Resultados: Se hallaron diferencias significativas en los estilos educativos desde la perspectiva de los niños y adolescentes con sobrepeso y obesidad o normopeso. Discusión: La percepción de los hijos sobre el estilo educativo parental no se relacionó con el peso corporal de estos.
\end{abstract}

Palabras clave: Estilo parental; Sobrepeso; Obesidad infantil.

\begin{abstract}
Introduction: The increase in childhood overweight and obesity has become a global problem in physical and psychological health. This problem has multiple causes, including parental style. Objective: The present study attempted to identify a relationship between parental style and children's perceived own overweight and obesity. Material and Method: Variables' measurements included the Affect Scale (EA) and the Norms and Requirements Scale, responded by children and adolescents. A total of 149 teens aged 12 to 16 years participated, of whom 24 were overweight or obese. Results: The results revealed an absence of significant differences in parental styles from the children's perspective, comparing those with overweight and obesity with those of normal weight. Conclusion: the perception of the parental educational style is not related to the weight of children or adolescents.
\end{abstract}

Key words: Parental style; Overweight; Childhood obesity.

\footnotetext{
${ }^{1}$ Av. Siervo de la Nación 845, Lomas del Valle, 58170 Morelia, Mich., México, tel. (443)299- 77-41, correo electrónico: itmg_0112@hotmail.com. ${ }^{2}$ Facultad de Psicología, Gral. Francisco Villa 450, Dr. Miguel Silva González, 58110 Morelia, Mich., México, tel. (443)312-99-09, correo electrónico: fpadros@uoc.edu.
} 
Recibido: $01 / 08 / 2020$

Aceptado: 24/11/2020

\section{INTRODUCCIÓN}

$\mathrm{E}$ 1 sobrepeso y la obesidad infantil son problemas que pueden aparecer desde la infancia, los que al no manejarse adecuadamente y con el apoyo apropiado por parte de los padres, pueden prevalecer hasta la edad adulta; además, se pueden complicar con otras enfermedades comórbidas de índole fisiológica o psicológica, según el caso. Datos de la Encuesta Nacional de Salud y Nutrición (Instituto Nacional de Salud Pública y Secretaría de Salud, 2018) indican que uno de cada tres adolescentes de entre 12 y 19 años padece sobrepeso u obesidad, al igual que la de los niños en edad escolar (5 a 11 años) (cf. Romero et al., 2017).

La Organización Mundial de la Salud (OMS) (2016) define sobrepeso y la obesidad como una acumulación anormal o excesiva de grasa corporal que puede ser perjudicial para la salud y el Índice de Masa Corporal (IMC) es el principal indicador que se utiliza para determinar si una persona los padece, el cual se calcula dividiendo el peso de una persona en kilos por el cuadrado de su talla en metros $\left(\mathrm{kg} / \mathrm{m}^{2}\right)$; así, se considera sobrepeso cuando el IMC es igual o superior a 25 y obesidad cuando es igual o superior a 30 (OMS, 2016).

El sobrepeso y la obesidad son de origen multifactorial, pues no solo influye en ellos la cantidad de alimento a ingerir y el gasto energético, sino también la genética. Deben tenerse en cuenta los aspectos psicológicos, sociales y culturales de la persona debido a que inciden en la ingesta de alimentos y en el ejercicio físico (Domínguez, Olivares y Santos, 2008).

En México, la obesidad contribuye con un número cercano a 200 mil muertos por año al ser un importante factor de riesgo de padecer enfermedades crónico-degenerativas, como la diabetes mellitus tipo II, las enfermedades isquémicas del corazón, las cerebro-vasculares y las hipertensivas (Sánchez, Pichardo y López, 2004). Aunque no solo se asocia frecuentemente a este tipo de enfermedades, ya que, por otro lado, la persona con obe- sidad tiene mayor riesgo de padecer alteraciones psicopatológicas y trastornos del sueño (Tubbs, Khader, Fernandez y Grandner, 2020).

Para evitar el sobrepeso y la obesidad es conveniente que los niños adquieran buenos hábitos alimenticios como el comer sanamente (basadas en las 5 comidas y raciones sugeridas), tratar de evitar las comidas rápidas y la denominada "chatarra", etc. (Briones y Cantú, 2003). Kiefner, Hoffmann, Meers, Koball y Musher-Eisenman (2014) subrayan la relevancia que tienen los padres de familia en los comportamientos alimenticios de sus hijos y, por ende, en su peso corporal, como lo es el practicar el control o la alimentación instrumental (presión para comer, restricción de ciertos alimentos o empleo de alimentos como recompensa), suscitar el comer en exceso o dietas pobres, o fomentar hábitos que interfieren con la capacidad de los niños para un consumo de energía autorregulado.

Es importante mostrar que la paternidad es un factor social crítico en el desarrollo infantil y que abarca varios conceptos psicológicos, normas culturales y consideraciones prácticas (Treyvaud et al., 2016). Cabe señalar que el estilo parental también es concebido como un constructo multidimensional referente al tipo de relación entre padres e hijos, que incluye al menos ocho ejes principales (calidez parental, rechazo, estructura, caos, apoyo a la autonomía, coerción, exigencia y capacidad de respuesta) y tiende a ser estable (Baumrind, 1966; Skinner, Johnson y Snyder, 2005).

El contexto en el que los padres educan a sus hijos y sus estilos parentales -los cuales son el autoritario, el permisivo, el negligente y el democrático, según García, Cerezo, De la Torre, De Carpio y Casanova (2011) - tienen una estrecha relación con la forma de actuar, pensar y reaccionar de estos últimos. Según los autores citados, los estilos parentales inciden en el riesgo de alteraciones tales como la depresión, la ansiedad y la baja autoestima, así como de problemas para poner en práctica sus habilidades sociales con las demás personas. Por ello, se puede inferir que los estilos parentales pueden influir, por ejemplo, en la forma de comer de los hijos haciendo que aumenten la cantidad de comida que ingieren, que coman entre comidas, sin horarios, y sobre todo prefieran 
alimentos con altas cantidades de carbohidratos y grasas, sin poder autorregular sus impulsos por la comida en un intento por resolver sus problemas emocionales, lo que trae como resultado que los niños aumenten considerablemente de peso (cf. Contreras, 1995). En una revisión hecha por Danford, Schultz y Marvicsin (2015) se halló que el papel de los padres y su preocupación por la salud es fundamental en los comportamientos de alimentación y de actividad de sus hijos.

Los padres autoritarios o rígidos son poco afectuosos y muy controladores y exigentes. Las relaciones establecidas en las familias con padres autoritarios suelen ser frías, por lo que muy pocas veces se manifiestan sentimientos positivos y, por el contrario, es más común el rechazo de los padres hacia sus hijos, prohibiendo de diversas maneras la expresión de sentimientos de afecto, cariño y de tristeza inclusive, los que califican como propios de personas débiles. Algunas consecuencias en los niños de tal estilo parental que se han señalado son el riesgo de depresión infantil; el aislamiento del ambiente familiar, social y escolar, así como una baja autoestima y un escaso desarrollo de habilidades sociales y ansiedad, todos ellos inducidos por el temor a los castigos o a los propios padres, por lo que aquellos tratan de saciar esa necesidad comiendo (Iglesias y Romero, 2009; Montañés, Bartolomé, Montañés y Parra, 2008; Raya, 2008). En un estudio efectuado en Turquía por Yavuz y Selcuk (2018) se halló que la probabilidad de tener sobrepeso u obesidad era 4.71 veces mayor en los niños cuyas madres usaban un estilo de crianza autoritario, justo lo opuesto a lo encontrado por Tung y Yeh (2014), quienes observaron que los hijos con madres con estilos autoritario y democrático tenían un menor riesgo de padecer sobrepeso y obesidad. En una revisión llevada a cabo por Collins, Duncanson y Burrows (2014), los autores concluyen que un estilo de crianza autoritario se asocia con la presión para comer y manifestar comportamientos restrictivos de alimentación parental.

Los hijos de padres negligentes que sufren algún grado de obesidad, al no tener quien les exija ni los controle, hacen lo que quieren, comen lo que les apetece (cantidad y tipo de alimento) y a cualquier hora debido a que en la familia no existen reglas o normas que puedan favorecer la adopción de hábitos alimenticios saludables. Por ello, se ha señalado que la obesidad en las familias negligentes se debe a la falta de interés y de responsabilidad de los padres hacía sus hijos (Montañés et al., 2008; Raya, 2008).

En las familias con padres permisivos o indulgentes suele haber más respuestas del tipo afectuoso a las demandas de los hijos, por lo que sí se establecen normas en ellas. Sin embargo, los padres, con tal de que sus hijos se sientan bien, pueden mostrarse excesivamente permisivos e indulgentes con ellos. Por lo tanto, el niño con tendencia a la obesidad, al no tener unos padres que le exijan o pongan un alto a sus demandas en cuanto a la comida, puede comer lo que quiere sin restricción alguna. Tómese en cuenta que la capacidad del menor para autorregular su conducta no está muy desarrollada, por lo que come sin tener en cuenta las consecuencias que traerán consigo esos excesos (Iglesias y Romero, 2009; Montañés et al., 2008; Raya, 2008). Collins et al. (2014) señalan que un estilo de crianza permisivo está inversamente relacionado con el monitoreo de la ingesta alimentaria infantil. Shloim, Edelson, Martin y Hetherington (2015) han reportado asimismo un mayor riesgo de obesidad y sobrepeso en los hijos de padres permisivos.

Los padres democráticos o inductivos tienen el propósito de mantener de manera eficaz el bienestar de los miembros de su familia en todos los aspectos, lo que implica un cierto control sobre ellos, de sus conductas y hábitos a fin de que se garantice el mejor funcionamiento de la misma. La forma democrática de educar ayuda a los hijos a desarrollar de manera óptima la autorregulación de sus comportamientos, lo que hace posible que, con ayuda de sus padres y de su propio juicio, se cuiden de manera efectiva. Así, la obesidad puede considerarse un problema que requiere buscar maneras razonadas para evitarla o para regresar a un peso normal (Raya, 2008; Montañés et al., 2008; Iglesias y Romero, 2009). Shloim et al. (2015) afirman que hay una mayor probabilidad de que los hijos con padres democráticos tengan un peso saludable. 
En algunos estudios se ha encontrado que los hijos de madres autoritarias muestran mayor riesgo de sobrepeso que los hijos de aquellas con estilo democrático (Rhee, Lumeng, Appugliese, Kaciroti y Bradley, 2006; Ventura y Birch, 2008). Asimismo, se ha reportado que el estilo autoritario de ambos progenitores se relaciona positivamente con el riesgo de sufrir obesidad y sobrepeso (Luther, 2007; Vollmer y Mobley, 2013). Sin embargo, en un estudio llevado a cabo en Australia por Wake, Nicholson, Hardy y Smith (2007) no se observó ninguna relación entre el estilo de las madres y el sobrepeso de sus hijos. En la misma línea, Hughes, Shewchuk, Baskin y Nicklas (2008) y Olvera y Power (2010) han hallado que el estilo indulgente es el que se relaciona con mayor un IMC. Finalmente, Montiel y López (2017), en un estudio realizado con madres mexicanas, no encontraron relación entre la obesidad de los niños y algún estilo parental particular.

En los escasos estudios que toman en cuenta el estilo educativo del padre se ha observado que, en comparación con un estilo autoritario, los hijos de padres con estilos de crianza permisivos y negligentes tenían mayores probabilidades de cumplir con los criterios de sobrepeso u obesidad (Hughes et al., 2008; Wake et al., 2007), pero también se ha asociado un mayor riesgo de obesidad y sobrepeso con un padre autoritario (Luther, 2007; Vollmer y Mobley, 2013). Asimismo, también se ha observado que el estilo democrático de los padres funciona como un factor protector de sobrepeso y obesidad (Vollmer y Mobley, 2013). Debe señalarse que Mejia de Grubb et al. (2018) encontraron que los progenitores varones muestran una menor preocupación por el peso de sus hijos en comparación con las madres.

Vale mencionar que, en tales estudios, el estilo parental se determinó a partir de la información ofrecida por los propios padres, lo cual puede diferir considerablemente de la precepción que del mismo tienen sus hijos (Bersabé, Fuentes y Motrico, 2001). Por ello, se considera importante estudiar el estilo parental a partir de dicha percepción.

De este modo, se considera importante el estilo educativo debido a que, ya sea de manera autoritaria o permisiva, los padres son quienes eligen los alimentos de sus hijos, y quienes determi- nan el tipo, la cantidad y el momento en que se deben consumir. Por ello, se parte de la idea de que el estilo parental influye en lo que sus hijos comen y en la adopción de conductas alimentarias propias, como el rechazo o la aceptación a ciertos alimentos, e incluso la cantidad y el horario, lo que los lleva a una buena o mala mutrición, y por consiguiente a una buena salud o a la obesidad y el sobrepeso (Domínguez et al., 2008). De acuerdo con Kiefner-Burmeister et al. (2014), las prácticas alimentarias empleadas por los padres autoritarios y permisivos pueden conducir a sus hijos a tener dificultades para autorregular su ingesta de alimentos o a comer en ausencia de hambre. Es por ello que el objetivo principal de esta investigación fue analizar si hay una relación entre el estilo parental percibido por los hijos con el sobrepeso y la obesidad infantil en población mexicana.

\section{MÉTODO}

\section{Participantes}

Participó voluntariamente un total de 149 alumnos de sexto año de una escuela primaria y de una secundaria, con edades comprendidas entre $12 \mathrm{y}$ 16 años $(\mathrm{M}=13.36$; D.E. $=1.36)$, de los cuales 76 $(51.01 \%)$ eran de sexo masculino. Se utilizó como único criterio de inclusión que fueran alumnos de dichas escuelas.

\section{Instrumentos}

Escala de Afecto (EA-H) (Bersabé et al., 2001). Este instrumento evalúa comportamientos parentales como el afecto, la comunicación, la crítica y el interés hacia los hijos, y cómo estos perciben a sus padres en cuanto a los comportamientos que tienen hacia ellos. Se compone de dos subescalas: Afecto-comunicación, que determina cómo se manifiesta el afecto, la comunicación y el interés de los padres hacia sus hijos, y Crítica-rechazo, referida a la crítica, el rechazo y la falta de confianza de los padres en sus hijos. Los valores de la confiabilidad de los factores, medidos por el coeficiente alfa de Cronbach, oscilan entre .60 y 0.85 . 
Escala de Normas y Exigencias (ENE-H)

(Bersabé et al., 2001).

Evalúa los estilos educativos parentales. La perspectiva a considerar es cómo los hijos perciben a sus padres en cuanto a los comportamientos que tienen hacia ellos. Consta de tres subescalas: 1) Forma inductiva o democrática (padres que explican y toman en cuenta las necesidades de sus hijos al establecer las normas familiares y exigen su cumplimiento); 2) Forma rígida o autoritaria (padres que exigen el cumplimiento de las normas, con un alto nivel de control sobre sus hijos), y 3) Forma indulgente o permisiva (padres que aunque haya normas no las imponen ni exigen su cumplimiento). La interpretación es que entre mayor es la puntuación en cada subescala, más alta es la tendencia a desempeñar ese estilo educativo parental. Se ubica al padre en el estilo parental en el que haya obtenido la mayor puntuación, y en el caso de que haya obtenido puntuaciones semejantes, no se le ubica en ningún estilo concreto. La consistencia interna de cada uno de los factores, medida por el coeficiente alfa de Cronbach, varió entre 0.60 y 0.90 (Bersabé, Fuentes y Motrico, 2003). Se utilizó una adaptación a la población española en virtud de que no hay todavía una adaptación a la mexicana.

\section{Procedimiento}

En primer lugar, se solicitó que el proyecto fuera evaluado por el Comité de Ética de la Facultad de Psicología de la Universidad Michoacana, de San Nicolás de Hidalgo. Una vez obtenida la aprobación, se solicitó la autorización a las escuelas para aplicar el protocolo de la investigación. Los responsables de las mismas hicieron llegar a los padres la información relativa a su consentimiento informado y los formatos respectivos, que estos últimos devolvieron firmados una semana más tarde. Una vez asignados los grupos por cada institución, en la primera sesión se solicitó a los estudiantes su participación voluntaria en el estudio (ningún niño rehusó participar), y en un término aproximado de 30 a 45 minutos por grupo se aplicaron en un solo día las escalas EA-H y ENE-H a cada escuela. Una vez aplicadas las escalas, al siguiente día se procedió en una segunda sesión, de aproximadamente 30 minutos por grupo, a medir y a pesar a cada uno de los alumnos para calcular su IMC.

Para el análisis de los datos se utilizaron los descriptivos como media aritmética, desviación estándar, frecuencia, porcentaje, mediana y valor intercuartil. Asimismo, para la estadística inferencial se empleó la prueba de $X^{2}$, la $t$ de Student-Fisher para datos independientes, la prueba de Kolmogorov-Smirnov, la U de Mann-Whitney y la correlación de Pearson. El software utilizado para el análisis de datos fue el SPSS, versión 20.0, y el paquete AMOS, versión 22, de Windows.

\section{RESULTADOS}

Los datos de los participantes fueron los siguientes; sobre la estatura $(\mathrm{M}=1.55$; $\mathrm{D}, \mathrm{E} .=0.08)$; el peso $(\mathrm{M}=50.70 \mathrm{k}$, D.E. $=11.30 \mathrm{k})$, y respecto al IMC $(M=20.89$; D.E. = 3.95). De los participantes, 21 de ellos (14.0\%) exhibían sobrepeso; 3 (2.0\%) obesidad y $125(83.3 \%)$, la mayoría, normopeso.

Respecto al sexo, 60 participantes en el grupo de normopeso (48\%) eran de sexo masculino y $65(52 \%)$ del femenino, y en el grupo de sobrepeso/obesidad, de 14 (58.3\%) niños y 10 (41.7\%) niñas. No se observaron diferencias significativas respecto a tal distribución entre ambas muestras $\left(X_{(1)}^{2}=0.860, p=0.354\right)$. En cuanto a la edad, en el grupo de normopeso, la media fue de 13.34 (D.E. $=1.33)$, y en el de sobrepeso/obesidad, de 13.50 (D.E. $=1.53$ ), sin diferencias significativas entre ellos $\left(t_{[147]}=-0.541, p=0.589\right)$.

En relación a la percepción del estilo educativo parental realizado por los hijos, tanto respecto a sus madres como a sus padres, se obtuvo que la mayoría de los hijos percibían a sus progenitores con un estilo inductivo (Tabla 1). No hubo diferencias significativas en la percepción del estilo de las madres $\left(X_{(2)}^{2}=2.086 ; p=0.352\right)$ ni de los padres $\left(X_{(2)}^{2}=2.717 ; p=0.257\right)$ respecto al normopeso y el sobrepeso/obesidad.

En primera instancia, se aplicó la prueba de Kolmogorov-Smirnov, observándose que ninguna de las variables se distribuía de forma acorde con la normalidad, por lo que se decidió aplicar pruebas no paramétricas. 
Tabla 1. Percepción de los hijos con normopeso y con sobrepeso/obesidad respecto al estilo educativo adoptado por sus padres.

\begin{tabular}{|l|c|c|c|}
\hline \multicolumn{1}{|c|}{ Padres } & Inductivo/democrático & Rígido/autoritario & Indulgente/permisivo \\
\hline Normopeso & $81(72.3 \%)$ & $21(18.8 \%)$ & $10(8.9 \%)$ \\
\hline Sobrepeso/obesidad & $15(71.4 \%)$ & $6(28.6 \%)$ & $0(0 \%)$ \\
\hline \multicolumn{1}{|c|}{ Madres } & Inductivo/democrático & Rígido/autoritario & Indulgente/permisivo \\
\hline Normopeso & $90(76.9 \%)$ & $18(15.4 \%)$ & $9(7.7 \%)$ \\
\hline Sobrepeso/obesidad & $20(87.0 \%)$ & $3(13.0 \%)$ & $0(0 \%)$ \\
\hline
\end{tabular}

Sobre los datos referidos a las escalas de afecto (EA) acerca de la percepción del hijo hacia el padre, no se observaron diferencias significativas en las subescalas de Afecto/comunicación y de Crítica/rechazo, ni tampoco respecto a la percepción del niño hacia la madre (Tabla 2).

Tabla 2. Percepción de los hijos hacía su padre y su madre en la Escala de Afecto (EA).

\begin{tabular}{|l|c|c|c|r|c|}
\hline \multirow{2}{*}{ ESCALA } & \multicolumn{2}{|c|}{ Normopeso } & \multicolumn{2}{c|}{ Sobrepeso/obesidad } & $\begin{array}{c}\text { U } \\
\text { de Mann- } \\
\text { Whitney }\end{array}$ \\
\cline { 2 - 6 } & M. y D.E. & Md y IQ & M. y D.E. & Md y IQ & $p$ \\
\hline EA1-HP & $34.95(9.43)$ & $35(30 \div 42)$ & $32.32(11.24)$ & $32.5(22.5 \div 42)$ & .296 \\
\hline EA2-HP & $17.95(5.94)$ & $17(13 \div 23)$ & $18.64(5.94)$ & $17.5(13 \div 20.5)$ & .995 \\
\hline EA1-HM & $39.01(8.24)$ & $41(33 \div 46)$ & $38.91(9.45)$ & $43(31.5 \div 46)$ & .838 \\
\hline EA2-HM & $18.35(6.79)$ & $17(13 \div 24)$ & $16.63(5.49)$ & $15(15.5 \div 18.5)$ & .369 \\
\hline
\end{tabular}

Nota: Escala de Afecto/Comunicación (EA1) y Escala de Crítica/Rechazo (EA2), del hijo hacia el padre (HP) y hacia la madre (HM).

En cuanto a la Escala de Normas y Exigencias (ENE) de la percepción del niño hacia su padre, los resultados indican que no hubo diferencias significativas en las subescalas (Forma inductiva o democrática, Forma rígida o autoritaria y Forma indulgente o permisiva) entre ambas muestras (Tabla 3).

Tabla 3. Percepción de los hijos hacia su padre en la Escala de Normas y Exigencias (ENE).

\begin{tabular}{|l|c|c|c|c|c|}
\hline \multirow{2}{*}{ ESCALA } & \multicolumn{2}{|c|}{ Normopeso } & \multicolumn{2}{c|}{ Sobrepeso/obesidad } & $\begin{array}{c}\text { U } \\
\text { de Mann- } \\
\text { Whitney }\end{array}$ \\
\cline { 2 - 6 } & M. y D.E. & Md y IQ & M. y D.E. & Md y IQ & $p$ \\
\hline ENE-IND & $36.93(9.35)$ & $37(30 \div 45)$ & $36.23(11.36)$ & $39.5(28 \div 44.5)$ & .932 \\
\hline ENE-RIG & $28.43(6.98)$ & $29(23 \div 33)$ & $29.73(7.58)$ & $30(26 \div 33.75)$ & .400 \\
\hline ENE-INDUL & $18.17(5.28)$ & $18(14 \div 21)$ & $15.91(5.66)$ & $16(10 \div 22)$ & .139 \\
\hline
\end{tabular}

Nota: Escala de Normas y Exigencias (ENE), Forma inductiva o democrática (IND), Forma rígida (RIG) y Forma indulgente (INDUL) del hijo hacia el padre (HP).

Tampoco se encontraron tales diferencias en la percepción del niño hacia la madre con estilos democrático y rígido; sin embargo, se observó un menor nivel en el puntaje del estilo indulgente en la muestra con sobrepeso u obesidad comparada con la muestra de normopeso (Tabla 4). 
Tabla 4. Percepción de los hijos hacia su madre en la Escala de Normas y Exigencias (ENE).

\begin{tabular}{|l|c|c|c|c|c|}
\hline \multirow{2}{*}{ ESCALA } & \multicolumn{2}{|c|}{ Normopeso } & \multicolumn{2}{c|}{ Sobrepeso/obesidad } & $\begin{array}{c}\text { U } \\
\text { de Mann- } \\
\text { Whitney }\end{array}$ \\
\cline { 2 - 6 } & M. y D.E. & Md y IQ & M. y D.E. & Md y IQ & $p$ \\
\hline ENE-IND & $39.41(7.87)$ & $41(34 \div 46)$ & $39.70(8.74)$ & $43(38 \div 44)$ & .727 \\
\hline ENE-RIG & $30.07(6.93)$ & $30(24 \div 35)$ & $30.50(7.81)$ & $30.5(23.5 \div 37)$ & .854 \\
\hline ENE-INDUL & $18.51(5.44)$ & $18(14 \div 23)$ & $16.04(5.54)$ & $15(11 \div 19.5)$ & .038 \\
\hline
\end{tabular}

Nota: Escala de Normas y Exigencias (ENE), Forma inductiva (IND), Forma rígida (RIG) y Forma indulgente (INDUL) del hijo hacia la madre (HM).

Finalmente, se estudió la relación entre el IMC, el sexo y la edad. No se observaron diferencias $(t[145]=-0.649, p=0.518)$ cuando se realizó una comparación de medias en el IMC entre niños $(\mathrm{M}=20.73$; D.E. $=4.04)$ y niñas $(\mathrm{M}=21.15$; D.E. $=3.90)$, ni tampoco entre la edad y el IMC $(\mathrm{r}=0.083 ; p=0.317)$.

\section{DISCUSIÓN}

El objetivo del presente estudio fue analizar la posible relación entre el estilo parental, el sobrepeso y la obesidad infantil en población mexicana. Primeramente debe tomarse en cuenta que en ambas muestras (normopeso y sobrepeso/obesidad) los niños consideraron que sus padres eran afectuosos y comunicativos en su ambiente familiar y poco rechazantes y críticos con ellos. Dichos resultados difieren de los hallados por Bersabé et al. (2003), quienes hallaron que los hijos perciben a sus padres menos inductivos, y más indulgentes y rígidos al momento de establecer y exigir el cumplimiento de las normas. Nótese que el grado de afecto y comunicación recibido o percibido entre los miembros de familia puede ser un indicador de la calidad de las relaciones, o bien, por el contrario, su deficiencia puede ser un factor predisponente para la aparición de problemas o rupturas en dichas relaciones. García et al. (2011) encontraron que el rechazo y el exceso de control que los hijos perciben en sus padres pueden aumentar los comportamientos dañinos inaceptables por la propia familia y por la sociedad.

Respecto al estilo parental de la madre, solamente se observaron diferencias en el estilo indulgente. Los niños con normopeso percibieron

Instituto de Investigaciones Psicológicas - Universidad Veracruzana en menor medida a su madre con un estilo indulgente. Los resultados del presente trabajo son diferentes a los hallados en varios estudios (Luther, 2007; Rhee et al., 2006; Tung y Yeh, 2014; Ventura y Birch, 2008; Vollmer y Mobley, 2013; Yavuz y Selcuk, 2018) en los que se ha reportado que los hijos de madres autoritarias tienen un mayor riesgo de sobrepeso que los niños de aquellas que mostraban un estilo democrático. Tales resultados son contrarios a los de Rhee et al. (2006), quienes hallaron que los hijos de madres permisivas y negligentes mostraban hasta el doble de probabilidad de tener un hijo con sobrepeso, comparados con los niños con madres con estilo democrático. Tampoco coinciden con los resultados de Shloim et al. (2015), que demuestran un mayor riesgo de obesidad infantil en los hijos de padres permisivos, y que los padres democráticos constituyen un factor de protección. En su estudio, Tung y Yeh (2014) encontraron que las madres con estilo autoritario y democrático eran un factor protector.

Los resultados del presente trabajo coinciden con los reportados por Olvera y Power (2010) y por Hughes et al. (2008) que hallaron que el estilo indulgente se relacionaba con mayor IMC; aunque no coinciden con el estudio realizado con población mexicana de Montiel y López (2017) que no encontraron diferencias significativas entre los estilos parentales de las madres de niños obesos respecto a las madres de niños con normopeso.

Por otro lado, no se hallaron diferencias en función de la percepción que tiene el hijo/a sobre su padre. Dichos resultados difieren de los de Hughes et al. (2008); de Wake et al. (2007), en los que los hijos de padres con estilos de crianza permisivos y negligentes mostraban una mayor probabilidad de sufrir sobrepeso u obesidad; de los 
de Luther (2007) y de los de Vollmer y Mobley (2013), que observaron una relación positiva entre el estilo autoritario del padre y la obesidad y sobrepeso. En esta investigación tampoco se apreció que el estilo democrático de los padres se relacionara de forma negativa con el sobrepeso y la obesidad (Vollmer y Mobley, 2013).

Las diferencias encontradas en el presente estudio y los previos, llevados a cabo en otros países, sugieren que los factores que tienen una mayor incidencia en la obesidad y el sobrepeso infantil en México son de otro tipo. Es posible que, socialmente, los progenitores no hayan asumido los hábitos alimentarios como un aspecto importante y que debe cuidarse en la educación de los hijos. Incluso podría suceder que la mayoría de población mexicana no perciba como amenazas la obesidad y sobrepeso, a diferencia de lo que ocurre en otros países. Debe señalarse que el estilo educativo parental puede ser distinto en función del área o aspecto que se aborde. Por ejemplo, se puede ser autoritario en el ámbito de los estudios y negligente respecto a los hábitos alimenticios (sobre todo si el progenitor no los percibe como relevantes). Debe recordarse que en el análisis de Danford et al. (2015) se señalaba como fundamental la preocupación por la salud de los padres; si estos no consideran como riesgo la obesidad o el sobrepeso de sus hijos, no incidirán en los comportamientos alimentarios ni en la actividad física de los mismos.

Cabe señalar que no obstante el aumento de los índices de sobrepeso y obesidad infantil, solamente $15 \%$ de los progenitores los consideran como un problema (Baughcum, Burklow, Deeks, Powers y Whitaker, 2001; Young-Hyman, Herman, Scott, Schlundt, 2000), pero no como un riesgo para su salud física (Adams, Quinn y Prince, 2005; Ortega, Rodríguez, González y Reyes, 2018).

Deben descartarse el sexo y la edad como variables extrañas que hayan podido incidir en los resultados del presente estudio, toda vez que no se encontraron diferencias en la distribución por sexo, ni en las medias de edad entre los menores con normopeso y aquellos con sobrepeso u obesidad. Tampoco se observó una relación entre el sexo y el IMC, ni entre este y la edad.

Es importante señalar algunas limitaciones que obligan a tomar con cautela estos resultados: en primer término, el número de participantes con obesidad y sobrepeso fue reducido, por lo que sería conveniente aumentar la muestra. Además, la muestra fue conformada de forma incidental y en un solo estado del país. Por tanto, sería deseable hacerlo de forma aleatoria y en diferentes estados. Otra limitación evidente es que las escalas de Bersabé et al. (2001) no han sido validadas para población mexicana, razón por la cual sería importante hacer uso de instrumentos psicométricos adaptados a la población a estudiar.

Debe tomarse en consideración que es una fortaleza el hecho de utilizar al niño como fuente de información, ya que su perspectiva es muy relevante porque es sobre su comportamiento que se puede incidir. Es posible aceptar que el niño o el adolescente sean informantes de dudosa credibilidad para valorar los estilos educativos parentales; por consiguiente, es necesario tomar con mucha precaución sus opiniones. Aunque la población de adultos no está exenta de sesgos - a estudiar por ejemplo el de la deseabilidad social-, sería deseable en futuras investigaciones tomar dicha información de fuentes diversas.

En conclusión, la mayoría de los niños percibió a sus madres y padres con un estilo parental inductivo o democrático, seguidos del rígido $\mathrm{y}$, por último, del indulgente. Respecto al objetivo principal, los resultados de este trabajo indican que la percepción del hijo sobre el estilo educativo parental no se relaciona con el peso de los niños ni de los adolescentes.

Citación: Mejía G., I.T. y Padrón B., F. (2022). ¿Influye el estilo parental percibido por el hijo en el sobrepeso u obesidad infantil? Psicología y Salud, 32(1), 151-160. https:// Doi.org/10.25009/pys.v32i1.2721. 


\section{REFRENCIAS}

Adams, A.K., Quinn, R.A. y Prince, R.J. (2005). Low recognition of childhood overweight and disease risk among native-American caregivers. Obesity Research, 13(1), 146-52. https://Doi.org/10.1038/oby.2005.19.

Baughcum, A.E., Burklow, K.A., Deeks, C.M., Powers, S.W. y Whitaker, R.C. (1998). Maternal feeding practices and childhood obesity. Archives of Pediatrics \& Adolescent Medicine, 152(10), 1010-1014. Doi: 10.1001/archpedi.152.10.1010.

Baumrind, D. (1966). Effects of authoritative parental control on child behavior. Child Development, 37(4), 887-907. Doi: $10.2307 / 1126611$.

Bersabé, R., Fuentes, M.J. y Motrico, E. (2001). Análisis psicométrico de dos escalas para evaluar estilos educativos parentales. Psicothema, 13(4), 678-684. Recuperado de https://www.redalyc.org/pdf/727/72713422.pdf.

Bersabé, R., Fuentes, M.J. y Motrico, E. (2003). Estrategias de socialización de los padres y conflictos entre padres e hijos en la adolescencia. Anuario de Psicología, 34(3), 385-400. Recuperado de https://revistes.ub.edu/index.php/Anuario-psicologia/article/viewFile/8739/10920.

Briones O., N. y Cantú P., M. (2004). Comparación diagnóstica de dos métodos antropométricos para la evaluación nutricional en preadolescentes del municipio de Guadalupe N.L., México. Revista de Salud Pública y Nutrición, 5(4). Recuperado de http://www.respyn.uanl.mx/v/4/index.html.

Collins, C., Duncanson, K. y Burrows, T. (2014). A systematic review investigating associations between parenting style and child feeding behaviours. Journal of Human Nutrition and Dietetics, 27(6), 557-568. Doi: 10.1111/jhn.12192.

Contreras, J. (1995). Alimentación y cultura: necesidades, gustos y costumbres. Barcelona: Edicions Universitat de Barcelona.

Danford, C.A., Schultz, C. y Marvicsin, D. (2015). Parental roles in the development of obesity in children: challenges and opportunities. Research and Reports in Biology, 6, 39-53. Doi: 10.2147/rrb.s75369.

Domínguez V., P., Olivares, S. y Santos J., L. (2008). Influencia familiar sobre la conducta alimentaria y su relación con la obesidad infantil. Archivos Latinoamericanos de Nutrición, 58(3) 249-255. Recuperado de http://repositorio.uchile.cl/bitstream/ handle/2250/123925/Dominguez_p.pdf?se.

García L., M.C., Cerezo M., T., De la Torre C., M.J., De Carpio F., M.V. y Casanova A., P.F. (2011). Prácticas educativas paternas y problemas internalizantes y externalizantes en adolescentes españoles. Psicothema, 23(4), 654-659. Recuperado de https://www.unioviedo.net/reunido/index.php/PST/article/viewFile/9140/9004.

Hughes, S.O., Shewchuk, R.M., Baskin, M.L., Nicklas, T.A. y Qu, H. (2008). Indulgent feeding style and children's weight status in preschool. Journal of Developmental and Behavioral Pediatrics, 29(5), 403-410. Doi: 10.1097/DBP.0b013e318182a976.

Iglesias, B. y Romero, E. (2009). Estilos parentales percibidos, psicopatológicos y personalidad en la adolescencia. Revista de Psicopatología y Psicología Clínica, 14(2), 63-77. Doi: 10.5944/rppc.vol.14.num.2.2009.4067.

Instituto Nacional de Salud Pública y Secretaría de Salud (2018). Encuesta Nacional de Salud y Nutrición 2018. Ciudad de México: Autores.

Kiefner-Burmeister, A., Hoffmann, D.A., Meers, M.R., Koball, A.M. y Musher-Eisenman, D.R. (2014). Food consumption by young children: A function of parental goals and practices. Appetite, 74, 6-11.

Luther, B. (2007). Looking at childhood obesity through the lens of Baumrind's parenting typologies. Orthopaedic Nursing, 26(5) 270-278. Doi: 10.1097/01.NOR.0000295951.97129.3f.

Mejia de G., M.C., Salemi J., L., Gonzalez S., J., Sanderson, M., Zoorob, R.J., Mkanta, W. y Levine, R.S. (2018). Parenting style and perceptions of children's weight among US Hispanics: a qualitative analysis. Health Promotion International, 33(1), 132-139. Doi: 10.1093/heapro/daw050.

Montañés, M., Bartolomé, R., Montañés, J. y Parra, M. (2008). Influencia del contexto familiar en las conductas adolescentes. Ensayos, 17, 391-407. Recuperado de http://www.uclm.es/ab/educacion/ensayos/pdf/revista23/23_20.pdf.

Montiel M., M. y López L., F. (2017). Parenting styles and their relation with obesity in children ages 2 to 8 years. Revista Mexicana de Trastornos Alimentarios, 8(1), 11-20. Doi: 10.1016/j.rmta.2017.01.006.

Olvera, N. y Power, T. (2010). Brief report: Parenting styles and obesity in Mexican American children: A longitudinal study. Journal of Pediatric Psychology, 35(3), 243-249. Doi: 10.1093/jpepsy/jsp071.

Organización Mundial de la Salud (2016). Informe de la Comisión para acabar con la obesidad infantil. Ginebra: OMS. Recuperado de http://apps.who.int/iris/bitstream/10665/206450/1/9789243510064_spa.pdf?ua $=1$.

Ortega A., D.V., Rodríguez O., G., González U., M.A. y Reyes M., H. (2018). Perceptions of childcare staff for preventing overweight in Mexican preschool children: A swot analysis. Salud Pública de México, 60(2), 166. Doi: 10.21149/8897.

Raya A., F. (2008). Estudio sobre los estilos educativos parentales y su relación con los trastornos de conducta en la infancia. Tesis doctoral inédita. Córdoba (España): Universidad de Córdoba). Recuperado de http://helvia.uco.es/xmlui/bitstream/ handle/10396/2351/abre_fichero.pdf? sequence $=1$. 
Rhee, K.E., Lumeng, J.C., Appugliese, D.P., Kaciroti, N. y Bradley, R.H. (2006). Parenting styles and overweight status in first grade. Pediatrics, 117(6), 2047-2054. Doi: 10.1542/peds.2005-2259.

Romero M., M., Shamah L., T., Cuevas N., L., Gómez H., I.M., Gaona P., E.B., Gómez A., L.M., Rivera D., J.A y Hernández Á., M. (2017). Diseño metodológico de la Encuesta Nacional de Salud y Nutrición de Medio Camino 2016. Salud Pública de México, 59, 299-305. Recuperado de https://www.scielosp.org/article/spm/2017.v59n3/299-305/es/.

Sánchez C., C.P., Pichardo O., E. y López, P. (2004). Epidemiología de la obesidad. Gaceta Médica de México, 140(2), 3-20.

Shloim, N., Edelson, L.R., Martin, N. y Hetherington, M.M. (2015). Parenting styles, feeding styles, feeding practices, and weight status in 4-12 year-old children: a systematic review of the literature. Frontiers in Psychology, 6, 1849. Doi: 10.3389/fpsyg.2015.01849.

Skinner, E., Johnson, S. y Snyder, T. (2005). Six dimensions of parenting: A motivational model. Parenting: Science and Practice, 5(2), 175-235. Doi: 10.1207/s15327922par0502_3.

Treyvaud, K., Doyle, L.W., Lee, K.J., Ure, A., Inder, T.E., Hunt, R.W. y Anderson, P.J. (2016). Parenting behavior at 2 years predicts school-age performance at 7 years in very preterm children. Journal of Child Psychology and Psychiatry, 57(7), 814-821. Doi: 10.1111/jcpp.12489.

Tubbs, A.S., Khader, W., Fernandez, F. y Grandner, M.A. (2020). The common denominators of sleep, obesity, and psychopathology. Current Opinion in Psychology, 34, 84-88. Doi: 10.1016/j.copsyc.2019.11.003.

Tung, H.J. y Yeh, M.C. (2014). Parenting style and child-feeding behaviour in predicting children's weight status change in Taiwan. Public Health Nutrition, 17(5), 970-978. Doi: 10.1017/s1368980012005502.

Ventura, A.K. y Birch, L.L. (2008). Does parenting affect children's eating and weight status? International Journal of Behavioral Nutrition and Physical Activity, 5(1), 1-12. Doi: 10.1186/1479-5868-5-15.

Vollmer, R.L. y Mobley, A.R. (2013). Parenting styles, feeding styles, and their influence on child obesogenic behaviors and body weight. A review. Appetite, 71, 232-241. Doi: 10.1016/j.appet.2013.08.015.

Wake, M., Nicholson, J.M., Hardy, P. y Smith, K. (2007). Preschooler obesity and parenting styles of mothers and fathers: Australian national population study. Pediatrics, 120(6), e1520-e1527. Doi: 10.1542/peds.2006-3707.

Yavuz, H.M. y Selcuk, B. (2018). Predictors of obesity and overweight in preschoolers: The role of parenting styles and feeding practices. Appetite, 120, 491-499. Doi: 10.1016/j.appet.2017.10.001.

Young-Hyman, D., Herman, L.J., Scott, D.L. y Schlundt, D.G. (2000). Caregiver perception of children's obesity-related health risk: A study of African American families. Obesity Research, 8(3), 241-248. Doi: 10.1038/oby.2000.28.

Instituto de Investigaciones Psicológicas - Universidad Veracruzana

ISSN impreso: 1405-1109
Psicología y Salud, Vol. 32, Núm. 1: 151-160, enero-junio de 2022 https://doi.org/10.25009/pys.v32i1.2721 